\title{
Three-dimensional theory on supercavitating hydrofoils near a free surface
}

\author{
By OKITSUGU FURUYA \\ Division of Engineering and Applied Science, California Institute of Technology $\dagger$
}

(Received 2 December 1974)

Supercavitating hydrofoils of large aspect ratio operating near a free surface are investigated, assuming an inviscid and irrotational flow with the effects of gravity and surface tension neglected. The flow near the foil, treated as twodimensional, is solved by a nonlinear free-streamline theory, then a threedimensional 'downwash' correction is made using Prandtl's lifting-line theory. The strength of the lifting-line vortex is determined by information from the twodimensional solution through a matching procedure, in which the inverse of aspect ratio is used as a small parameter for asymptotic expansions. The analysis incorporates a free-surface reference level to determine the submergence depth of the foil. The present method can be applied to any type of foil having an arbitrary planform or profile shape, including a rounded leading edge, a twist and even a small dihedral angle, within the assumption of large aspect ratio. Numerical computations made on rectangular flat-plate hydrofoils show excellent agreement of results with existing experimental data, even for large angles of attack and relatively low aspect ratios. The pressure distributions, shapes of the cavity and free surface are also calculated as a function of spanwise position.

\section{Introduction}

The idea of using a hydrofoil as a lifting device for high-speed hydrofoil craft has a long history, going back well into the nineteenth century (see e.g. Acosta 1973). It is, however, only in recent years that high cruising speeds, say above 60 knots, have been sought, resulting in the inevitable cavitation on the foil. There has been no simple yet accurate method available to date that can properly incorporate the complete features of the title problem such as finite aspect ratio, submergence depth of the foil, arbitrary foil and planform shapes, a spanwise twist, dihedral angle, and so on. To take into account the three-dimensional effect, Nishiyama (1970) and Leehey (1971) used Prandtl's lifting-line theory, and Widnall (1966) and Nishiyama \& Miyamoto (1969) used a lifting-surface theory. All of them are fully linearized theories, in terms of the body angle, however, naturally setting a limit on the application of their methods to only the flow at small angles of attack and small cavitation numbers.

The objective of the present paper is, therefore, to provide a simple yet

$\dagger$ Present Address: Tetra Tech, Inc., Pasadena, California 91107, U.S.A. 
accurate method for design of supercavitating hydrofoils of large aspect ratio near a free surface, having practically no limitations on the admissible foil profile and angle of attack. The present method consists essentially of two parts, one being a two-dimensional nonlinear free-streamline theory used for the near-field flow region, and the other being Prandtl's lifting-line theory for the far-field flow region. The definition of the two flow regions will be made by introducing a small parameter, $\epsilon \equiv 1 /$ (aspect ratio), where (aspect ratio) $=(\text { span })^{2} /$ (projected area of foil); the near field is the flow region whose distance from the position of the foil is comparable to the chord length whereas the far field is that at the span length. The near-field or inner solution, which is asymptotically valid as $\epsilon \rightarrow 0$ with the chord length fixed, is obtained by a two-dimensional free-streamline theory. It is obviously of a great advantage to use a nonlinear theory, if possible, for this purpose, as was suggested by Shen \& Ogilvie (1972) in their work on planing surfaces of finite span. The use of nonlinear free-streamline theory was made much easier by Furuya (1975), in which the flow around a supercavitating arbitrary foil near a free surface is exactly and directly solved with the foil shape and cavitation number specified. A foil of large aspect ratio, seen from far away, is observed to shrink down to just a lifting line. The far-field or outer solution as $\epsilon \rightarrow 0$ with the span fixed may, therefore, be represented by Prandtl's lifting-line theory. But the strength of this vortex line is unknown, since the detailed flow around the foil cannot be seen from the far field. The information will, however, be provided to determine the vortex strength through a 'matching' procedure. Usually, the matching procedure is carried out by expanding the inner and outer solutions in terms of the outer and inner co-ordinates, then blending them smoothly. (See e.g. Van Dyke 1964a; Cole 1968, for a general idea of perturbation theory and matching procedures.)

It must be noted that the potential of lifting-line theory remains valid for any flow angle or body angle $\alpha$ as long as the aspect ratio is assumed to be large. This is in contrast to the works mentioned above, in particular to that of Leehey, in which higher-order correction of aspect ratio was made, but within a small- $\alpha$ assumption.

This type of method was first applied by Van Dyke (1964b) to a fully wetted airfoil problem in an unbounded flow medium, and recently by Shen \& Ogilvie (1972) to finite span flat-plate planing surfaces. In particular, the latter is similar to the present problem, in that a planing surface of deep submergence is equivalent to a limiting case of the cavity flow or infinite cavity flow, and also in that the reference level of water must be properly incorporated into the analysis in spite of neglecting the gravity effect. As is discussed at considerable length by Shen \& Ogilvie, it is a well-known fact that any two-dimensional analysis on this type of free-surface problem creates non-unique behaviour of the free surface location at infinity, owing to the exclusion of the gravity effect. (The free surface falls off logarithmically and does not reach a definite reference level.) This was implicitly described by Green (1936), in solving the problem of a two-dimensional gliding plate on a water surface. One of the methods of representing this free-surface flow in a physically acceptable way is to use a three-dimensional feature for the far-field solution, as was pointed out by Shen \& Ogilvie. With this, the free-stream- 


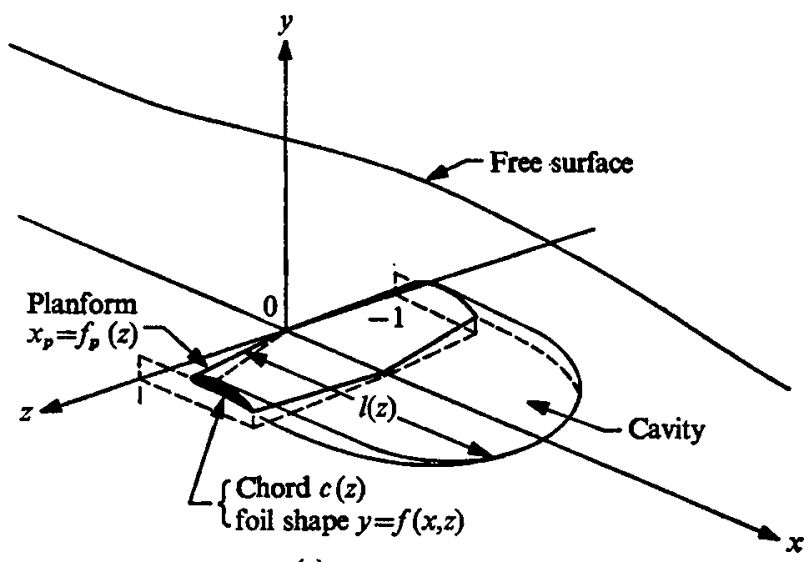

(a)

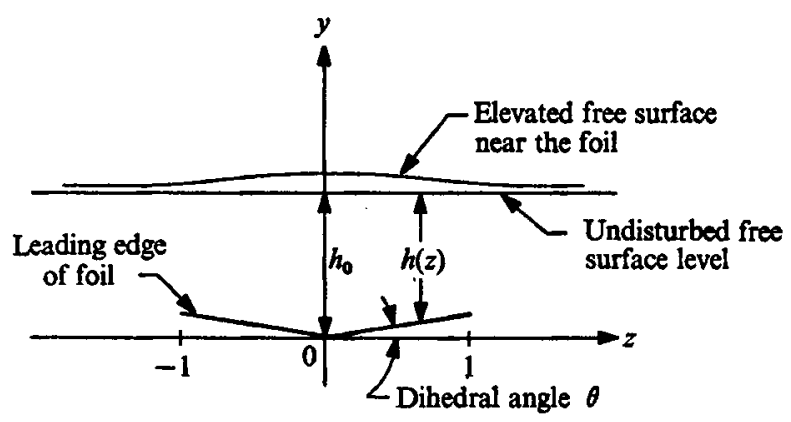

(b)

Figure 1. (a) Flow configuration. (b) A view from upstream infinity, showing the relation between a dihedral angle $\theta_{D}$ and submergence depth $h(z)$.

line elevation dies off to a definite reference level and therefore the foil submergence $h$ under an undisturbed water level can be determined uniquely. But it is noted that the present method uses a somewhat different technique from that of Shen \& Ogilvie, which is more appropriate for the present problem, as will be seen later.

\section{Mathematical formulation}

The problem treated here is flow around a three-dimensional supercavitating foil of large aspect ratio operating near a free surface, as is depicted in figure 1 , in which a right-handed co-ordinate system is used with the origin fixed at the midspan leading edge. It is always convenient to choose a normalized length, so that the half-span is taken to be unity in the present problem. A complete foil geometry is specified by giving the leading-edge planform $x_{p}=f_{p}(z)$, foil camber shape $y=f(x, z)$, chord length $c(z)$ and a dihedral angle of the foil. The dihedral angle is presently given by specifying the foil submergence depth $h(z)$, where $h(z)$ is the distance between the $y$ co-ordinate of the leading edge and that of the undisturbed free-surface level at infinity. For example, the specification of $h(z)=h_{0} \pm z \tan \theta_{D}$ 
gives a symmetric dihedral angle of $\theta_{D}$ to the leading edge, where $h_{0}$ is the submergence depth at the midspan. In the present problem, $h(z)$ is also assumed to be of the same order as the chord length, so that $h /(\operatorname{span})=O(\epsilon)$, too. In addition, the flow is assumed to be ideal, irrotational and gravity and surfacetension free.

\subsection{Far-field solution}

The far-field solution is the one obtained from the limiting case as $\epsilon \rightarrow 0$ with the span fixed. With $h$ assumed to be of order $\epsilon$, the foil observed from afar, comparable to the span length at least, is a lifting line lying on the free surface itself, with all detailed flow information invisible, as is suggested in figure 2. The first two leading terms of the velocity potential $\phi$, satisfying the Laplace equation $\nabla^{2} \phi=0$, are given by

$$
\phi=x+\frac{1}{2 \pi} \int_{-1}^{1} \frac{y \gamma\left(z^{\prime}\right) d z^{\prime}}{y^{2}+\left(z-z^{\prime}\right)^{2}}\left(1+\frac{x}{\left[x^{2}+y^{2}+\left(z-z^{\prime}\right)^{2}\right]^{\frac{1}{2}}}\right)+O\left(\epsilon^{2}\right),
$$

where this form is exactly the same as that of the fully wetted foil in Van Dyke $(1964 b)$, except that the factor in front of the second term is $\frac{1}{2} \pi$ instead of $\frac{1}{4} \pi$, here owing to an image effect of a free surface. The first term is, of course, the potential of an undisturbed flow where the velocity $q_{\infty}$ is normalized to be unity, and the second one is that due to the line vortex and its trailing vortices, with an as yet unknown vortex strength $\gamma(z)$. This second term has no velocity component in the $x$ direction, and therefore satisfies the dynamic boundary condition on the free surface (i.e. $p=p_{\infty}$ ) to first order in $\epsilon$. In addition, $\phi$ satisfies a radiation condition that the disturbance created by the body vanishes at upstream infinity.

\subsection{Near-field solution}

The near-field solution is to be found as the limiting case of $\epsilon \rightarrow 0$ with, this time, the chord length fixed. We first introduce by definition a cavitation number

$$
\sigma=\frac{p_{\infty}-p_{c}}{\frac{1}{2} \rho q_{\infty}^{2}}
$$

where $p_{\infty}$ and $p_{c}$ are the static pressures at upstream infinity and inside the cavity (assumed to be constant), $\rho$ is the density of the fluid medium and $q_{\infty}$ is the velocity at upstream infinity, normalized to unity. The foil geometry and corresponding boundary conditions, as well as the Laplace equation, are rewritten in a new co-ordinate system $(X, Y, Z) \equiv(x / \epsilon, y / \epsilon, z)$, to become more 'shortsighted' in the near-field flow region, the result being summarized in table 1. The boundary conditions on the body and cavity, which have never before appeared in the far-field region, are now incorporated in the inner solution.

For the cavity termination condition, the double-spiral vortex model of Tulin (1964) is employed, so that the pressure is assumed to jump from $p_{c}$ to $p_{\infty}$ at the end point of the cavity. The word wake denotes the region inside the two streamlines followed by the regions of the cavity boundaries. (See also Furuya 1975.) The first- and second-order solution for $\tilde{\Phi}$ should then satisfy (i) Laplace's equation

$$
\Phi_{X X}+\Phi_{Y Y}=0
$$




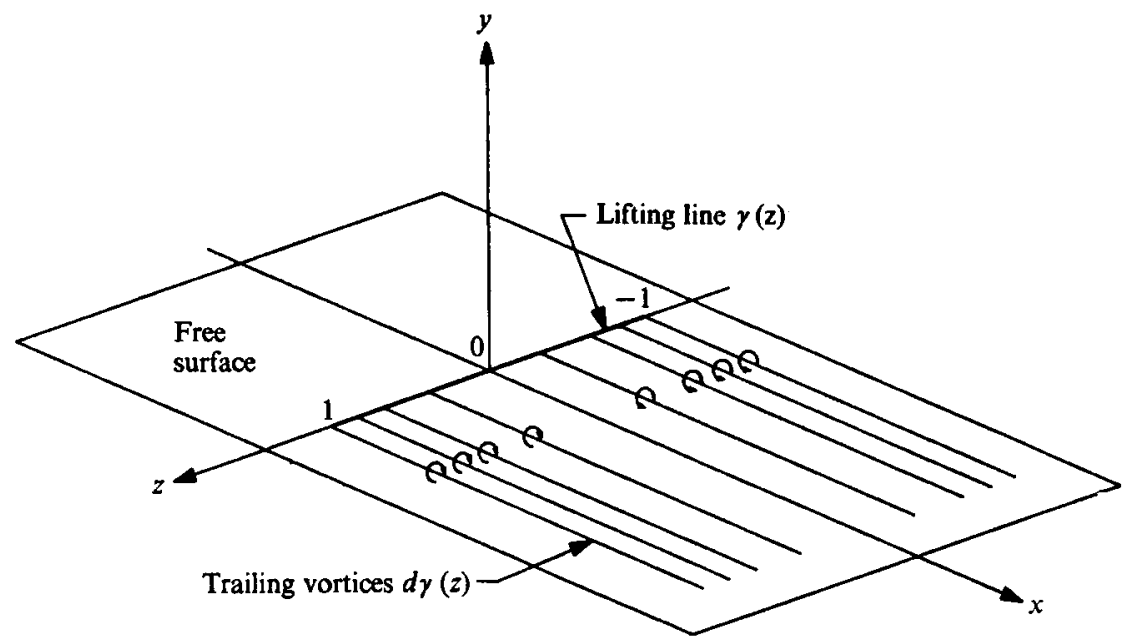

Figure 2. Lifting-line concept for the supercavitating foil of large aspect ratio, near a free surface, to be used for the far-field solution.

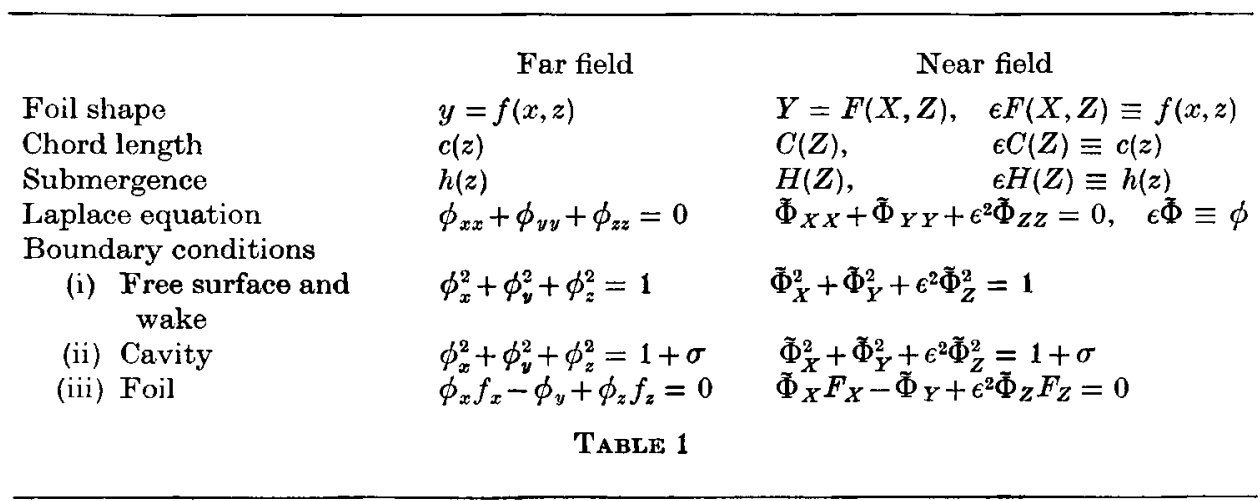

(ii) on the free surface and wake

(iii) on the cavity

$$
\Phi_{X}^{2}+\Phi_{Y}^{2}=1
$$

and (iv) on the body

$$
\begin{gathered}
\Phi_{X}^{2}+\Phi_{Y}^{2}=1+\sigma, \\
\Phi_{Y} / \Phi_{X}=F_{X},
\end{gathered}
$$

where $\Phi$ denotes the first- and second-order part of $\tilde{\Phi}$, so that $\tilde{\Phi}=\Phi+O\left(\epsilon^{2}\right)$. The flow here is taken to be completely two-dimensional, and the same as that of Furuya (1975), except that the information at upstream and downstream infinity is missing, and the previously specified parameter $\Psi_{0}$ becomes one of the unknown parameters, to be determined with $h$ specified. $\Psi_{0}$ here represents the total flow rate per unit span of the upper cavity sheet. The flow configuration is shown in figure 3 , in which the flow approaches the foil, and stagnates at a yet unknown stagnation point $S_{t}$, splitting into two parts, one going down along the lower part of the foil, and one going up along the upper surface. The cavity is then assumed to spring from the trailing edge $T$, and upper separation point $B$, both fixed but arbitrary, and to terminate at the points $A$ and $C$, followed by the wake downstream. 


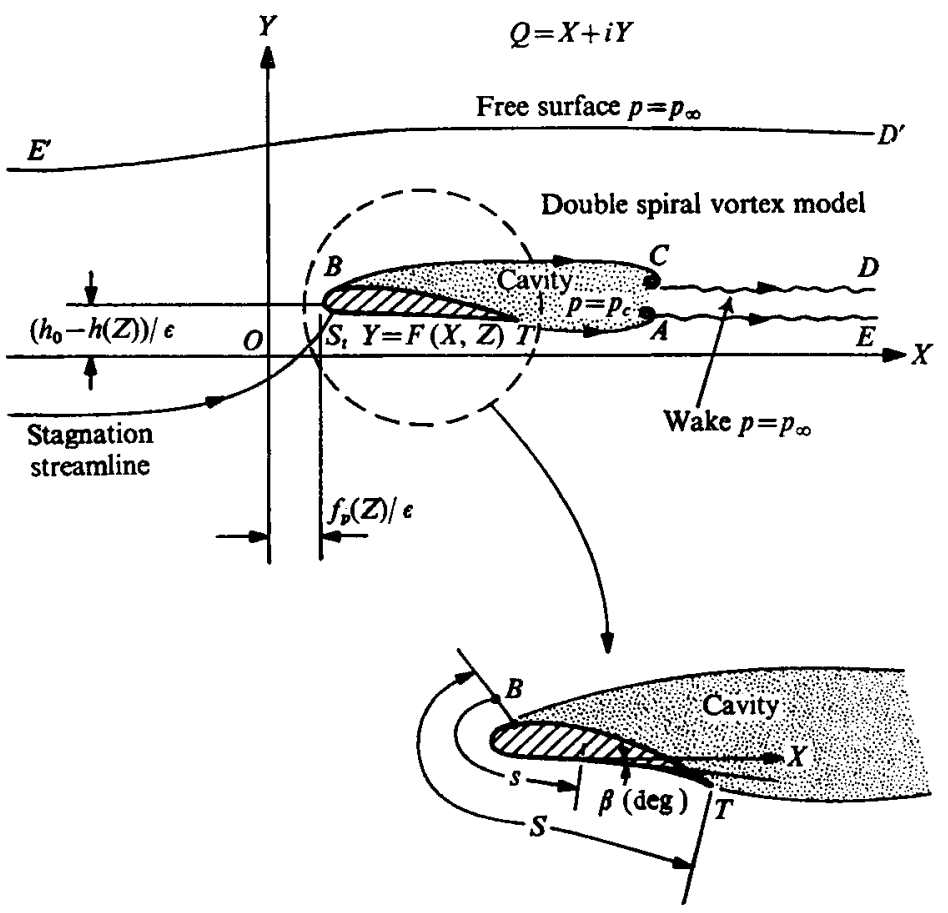

Figure 3. Two-dimensional sectional flow configuration in the expanded co-ordinate system $(X, Y) \equiv(x / \epsilon, y / \epsilon)$.

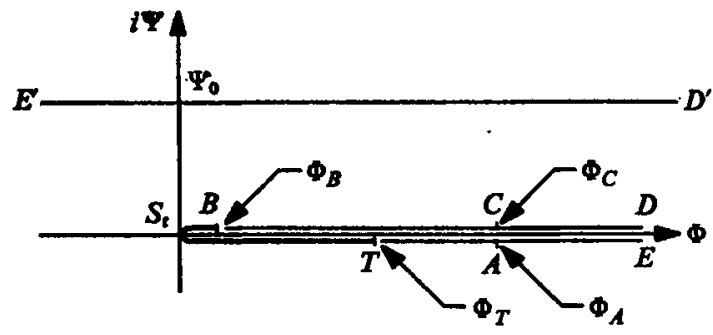

(a)

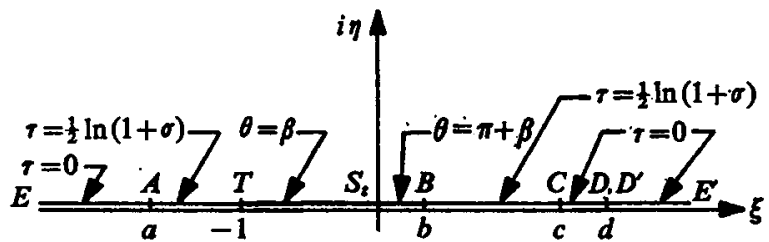

(b)

Figure 4. $(a)$ Potential $W=\Phi+i \Psi$ plane of the two-dimensional flow.

(b) Transform $\zeta$ plane, together with boundary conditions. 
Since the complex potential $W=\Phi+i \Psi$ exists in the near-field flow region with $W=0$ at the stagnation point, the physical plane $(X, Y)$ can be mapped onto the $W$ plane, as shown in figure $4(a)$. A new analytic function $\omega$ is now introduced, $\quad d W / d Q=q \exp (-i \theta)=q_{\infty} \exp (-i \omega), \quad Q=X+i Y$,

where $q$ is the magnitude of the flow velocity and $\theta$ is the flow angle, then

$$
\omega=\theta+i \tau, \quad \tau=\ln \left(q / q_{\infty}\right) .
$$

The $W$ plane is once more mapped onto the upper half of a new $\zeta=\xi+i \eta$ plane in figure $4(b)$ by the mapping function

$$
W=-\frac{\Psi_{0}}{\pi d}\left(\zeta+d \ln \frac{\zeta-d}{-d}\right)
$$

where $d$ is the $\xi$ co-ordinate corresponding to the point $D$ or $D^{\prime}$ at downstream infinity. All $\xi$ co-ordinates $a, b, c, d$, as well as $\Psi_{0}$, are as yet unknown parameters. All boundary conditions on the $\xi$ axis are expressed either in terms of $\theta$ or $\tau$ of (4), leading to a mixed-type boundary-value problem, whose solution is (see Furuya 1975)

$$
\begin{aligned}
\omega(\zeta)= & H(\zeta) G(\zeta)=[(\zeta+1)(\zeta-b)]^{\frac{1}{2}}\left\{\frac{1}{2 \pi i} \int_{a}^{-1} \frac{-i \ln (1+\sigma)}{\left[\left(\xi^{\prime}+1\right)\left(\xi^{\prime}-b\right)\right]^{\frac{1}{2}}} \frac{d \xi^{\prime}}{\xi^{\prime}-\zeta}\right. \\
& +\frac{1}{2 \pi i} \int_{-1}^{b} \frac{2 \beta\left(\xi^{\prime}\right)}{i\left[\left(1+\xi^{\prime}\right)\left(b-\xi^{\prime}\right)\right]^{\frac{1}{2}}} \frac{d \xi^{\prime}}{\xi^{\prime}-\zeta}+\frac{1}{2 \pi i} \int_{0}^{b} \frac{2 \pi}{i\left[\left(1+\xi^{\prime}\right)\left(b-\xi^{\prime}\right)\right]^{\frac{1}{2}}} \frac{d \xi^{\prime}}{\xi^{\prime}-\zeta} \\
& \left.+\frac{1}{2 \pi i} \int_{b}^{c} \frac{i \ln (1+\sigma)}{\left[\left(\xi^{\prime}+1\right)\left(\xi^{\prime}-b\right)\right]^{\frac{1}{2}}} \frac{d \xi^{\prime}}{\xi^{\prime}-\zeta}\right\},
\end{aligned}
$$

where $\beta=\tan ^{-1}(\partial F(X, Z) / \partial X\}$, and a Kutta condition has been applied at the trailing edge of the foil. Only two conditions are available to be applied to this near-field solution. First, at the end points of the cavity we impose a condition

$$
\Phi_{C}-\Phi_{A}=\Gamma(Z) \equiv \gamma(z) / \epsilon
$$

proposed by Furuya (1975) as a modification to the double spiral vortex model. $\dagger$ The advantage of this condition is that no net force is left over on the wake. This relation is written in terms of $\xi$ co-ordinates by using (5):

$$
f_{1} \equiv-\frac{\Psi_{0}}{\pi d}\left(\tau+d \ln \frac{c-d}{-d}\right)+\frac{\Psi_{0}}{\pi d}\left(a+d \ln \frac{a-d}{-d}\right)-\frac{\gamma(z)}{\epsilon}=0 .
$$

Second, a proper scaling between the physical plane and $\zeta$ plane is required. On the wetted surface of the foil,

$$
d Q / d s=\exp (i \beta)
$$

where $Q=X+i Y$ and $s$ is an arc length measured from the upper cavity separation point $B$ (see figure 3 ). Integration of (8) using (3) and (5) provides

$$
\begin{aligned}
s(\xi) & =\int_{b}^{\xi} \exp \left[-i \beta\left(\xi^{\prime}\right)\right] \frac{d Q}{d W} \frac{d W}{d \xi^{\prime}} d \xi^{\prime} \\
& =\int_{b}^{\xi} \exp \left[-i \beta\left(\xi^{\prime}\right)\right] \exp \left[i \omega\left(\xi^{\prime}\right)\right]\left(-\frac{\Psi_{0}^{\prime}}{d \pi} \frac{\xi^{\prime}}{\xi^{\prime}-d}\right) d \xi^{\prime} \\
& =-\int_{\xi}^{b} \tilde{h}\left(\xi^{\prime}, a, b, c\right) k\left(\xi^{\prime}, d, \Psi_{0}\right) d \xi^{\prime},
\end{aligned}
$$

$\dagger$ Usually, $\Phi_{C}-\Phi_{A}=0$ is employed. (See, e.g. Larock \& Street 1967.) A similar idea was proposed by Acosta (1968). 
where

$$
\tilde{h}(\xi, a, b, c)=\exp \{-\operatorname{Im}(\omega(\xi))\}, \quad-1<\xi<b,
$$

and

$$
\begin{aligned}
& \tilde{h}(\xi, a, b, c)=\left[\operatorname { e x p } \left\{-\frac{\ln (1+\sigma)}{2 \pi}\left(\pi+\sin ^{-1} \frac{(1+\xi)(a-b)+(\xi-b)(1+a)}{(\xi-a)(1+b)}\right.\right.\right. \\
& \left.+\sin ^{-1} \frac{(1+\xi)(c-b)+(\xi-b)(1+c)}{(c-\xi)(1+b)}\right)+\frac{[(1+\xi)(b-\xi)]^{\frac{1}{2}}}{\pi} \\
& \left.\left.\times \oint_{-1}^{b} \frac{\beta\left(\xi^{\prime}\right)}{\left[\left(1+\xi^{\prime}\right)\left(b-\xi^{\prime}\right)\right]^{\frac{1}{2}}} \frac{d \xi^{\prime}}{\xi^{\prime}-\xi}\right\}\right] \frac{2 b^{\frac{1}{2}}[(1+\xi)(b-\xi)]^{\frac{1}{2}}+\xi(b-1)+2 b}{-\xi(1+b)}, \\
& k\left(\xi, d, \Psi_{0}\right)=\frac{d W}{d \xi}=-\frac{\Psi_{0}}{d \pi} \frac{\xi}{\xi-d},
\end{aligned}
$$

and $\oint$ means the Cauchy principal value of the improper integral. Now the proper scaling can be achieved by requiring

$$
f_{2} \equiv s(-1)-S(z)=0
$$

where $S(z)$ denotes the total arc length of the wetted portion depicted in figure 3 .

With the five unknown parameters, $a$ to $d$ and $\Psi_{0}$, we have so far obtained only two equations, still requiring three more to determine them uniquely. Two of them will be provided by the far-field solution, through the matching procedure in relation to the flow angle of attack and submergence. The third will be given by an additional assumption on the flow angle of the cavity sheet.

\subsection{Matching of the far-field and near-field solutions}

One of the most common techniques in the matching procedure is to expand the far-field and near-field solutions in terms of their counterpart co-ordinates, and to match corresponding terms to each other. (See Van Dyke $(1964 a, b)$ and Shen \& Ogilvie (1972) for this type of matching.)

First, the expansion of the far-field solution with $x=\epsilon X$ and $y=\epsilon Y$ substituted into (1) yields

$$
\phi \equiv \epsilon \Phi \sim \epsilon X-\frac{\gamma(z)}{\pi} \tan ^{-1} \frac{Y}{X}-\frac{\epsilon Y}{2 \pi} \int_{-1}^{1} \frac{\partial \gamma\left(z^{\prime}\right) / \partial z^{\prime}}{z-z^{\prime}} d z^{\prime}+O\left(\epsilon^{3}\right),
$$

which is similar to the result obtained by Van Dyke (1964b). On the other hand, the expansion of the near-field solution is somewhat more complicated, as the solution is implicitly given in terms of $\omega$ as a function of $\zeta$. First of all, we need to obtain the relation between $Q$ and $\zeta$. Using (3) we find

$$
d Q=d W / d \zeta \exp [i \omega(\zeta)] d \zeta .
$$

For a large $\zeta, \omega(\zeta)$ in (6) and $d W / d \zeta$ obtained from (5) can be expressed as

$$
\omega(\zeta)=R_{0}+R_{1} \zeta^{-1}+O\left(\zeta^{-2}\right), \quad \zeta \rightarrow \infty
$$

where $\quad R_{0}=\frac{\ln (1+\sigma)}{2 \pi}\left\{\ln \frac{-(b+1)}{2[(a+1)(a-b)]^{\frac{1}{2}}+(a+1)+(a-b)}\right.$

$$
\begin{aligned}
& \left.+\ln \frac{b+1}{2[(c+1)(c-b)]^{\frac{1}{2}}+(c+1)+(c-b)}\right\} \\
& +\frac{1}{\pi} \int_{-1}^{b} \frac{\beta\left(\xi^{\prime}\right)}{\left[\left(1+\xi^{\prime}\right)\left(b-\xi^{\prime}\right)\right]^{\frac{1}{2}}} d \xi^{\prime}+\left(\frac{\pi}{2}-\sin ^{-1} \frac{1-b}{1+b}\right),
\end{aligned}
$$




$$
\begin{aligned}
& R_{1}=\frac{1-b}{2} R_{0}+\left[\frac{1}{\pi} \int_{-1}^{b} \frac{\xi^{\prime} \beta\left(\xi^{\prime}\right) d \xi^{\prime}}{\left[\left(1+\xi^{\prime}\right)\left(b-\xi^{\prime}\right)\right]^{\frac{1}{2}}}+\frac{\ln (1+\sigma)}{2 \pi}\left\{-[(a+1)(a-b)]^{\frac{1}{2}}\right.\right. \\
& \left.-[(c+1)(c-b)]^{\frac{1}{2}}\right\}+\frac{1-b}{2} \ln \left|\frac{a+\frac{1}{2}(1-b)+[(a+1)(a-b)]^{\frac{1}{2}}}{c+\frac{1}{2}(1-b)+[(c+1)(c-b)]^{\frac{1}{2}}}\right| \\
& \left.+\left\{b^{\frac{1}{2}}-\frac{1-b}{2}\left(\frac{\pi}{2}-\sin ^{-1} \frac{1-b}{1+b}\right)\right\}\right] \text {. } \\
& \frac{d W}{d \zeta}=-\frac{\Psi_{0}}{\pi d}\left(1+\frac{d}{\zeta}+O\left(\zeta^{-2}\right)\right), \quad \zeta \rightarrow \infty .
\end{aligned}
$$

and

Substitution of (15) and (17) in (14) provides

$$
\begin{aligned}
d Q & =-\frac{\Psi_{0}}{\pi d}\left(1+\frac{d}{\zeta}+O\left(\zeta^{-2}\right)\right) \exp \left\{i\left(R_{0}+R_{1} \zeta^{-1}+O\left(\zeta^{-2}\right)\right)\right\} \\
& =-\frac{\Psi_{0}}{\pi d} \exp \left(i R_{0}\right)\left\{1+\left(i R_{1}+d\right) \zeta^{-1}+O\left(\zeta^{-2}\right)\right\} d \zeta, \quad \zeta \rightarrow \infty
\end{aligned}
$$

Integrating (18), we find

$$
Q=-\frac{\Psi_{0}}{\pi d} \exp \left(i R_{0}\right)\left\{\zeta+\left(i R_{1}+d\right) \ln \zeta+O(1)\right\}, \quad \zeta \rightarrow \infty,
$$

then invert (19) to obtain

$$
\zeta=-\frac{\pi d}{\Psi_{0}} \exp \left(i R_{0}\right) Q-\left(i R_{1}+d\right) \ln Q+O(1), \quad Q \rightarrow \infty .
$$

This relation (20) is then substituted into (5), to find

$$
W=\exp \left(-i R_{0}\right) Q+i \frac{\Psi_{0}}{\pi d} R_{1} \ln Q+O(1), \quad \text { as } \quad Q \rightarrow \infty
$$

The real part of (21) provides an expansion of $\Phi$,

$$
\begin{aligned}
\Phi & =\operatorname{Re}\{W\} \\
& =X \cos R_{0}+Y \sin R_{0}-\frac{\Psi_{0}}{\pi d} R_{1} \tan ^{-1} \frac{Y}{X}+O(1), \quad Q \rightarrow \infty,
\end{aligned}
$$

which is to be matched with its counterpart (13) from the far-field solution, finding, to order $\epsilon^{2}$,

$$
\begin{gathered}
\gamma(z)=\frac{\epsilon \Psi_{0}}{d} R_{1} \\
f_{3} \equiv R_{0}-\alpha_{D}=0, \quad \alpha_{D} \equiv-\frac{1}{2 \pi} \int_{-1}^{1} \frac{\partial \gamma\left(z^{\prime}\right) / \partial z^{\prime}}{z-z^{\prime}} d z^{\prime}
\end{gathered}
$$

where $R_{0}$ and $R_{1}$ are given in $(16 a, b)$. The relations (23) and (24) determine the previously unknown strength of the vortex line in the far-field solution and the missing boundary condition at upstream infinity in the near-field solution.

It is interesting to note that, if $R_{0}=0$, this is the far-upstream boundary condition in the two-dimensional problem, denoting the zero-flow angle found in Furuya (1975). But, in the present three-dimensional analysis, (24) indicates 


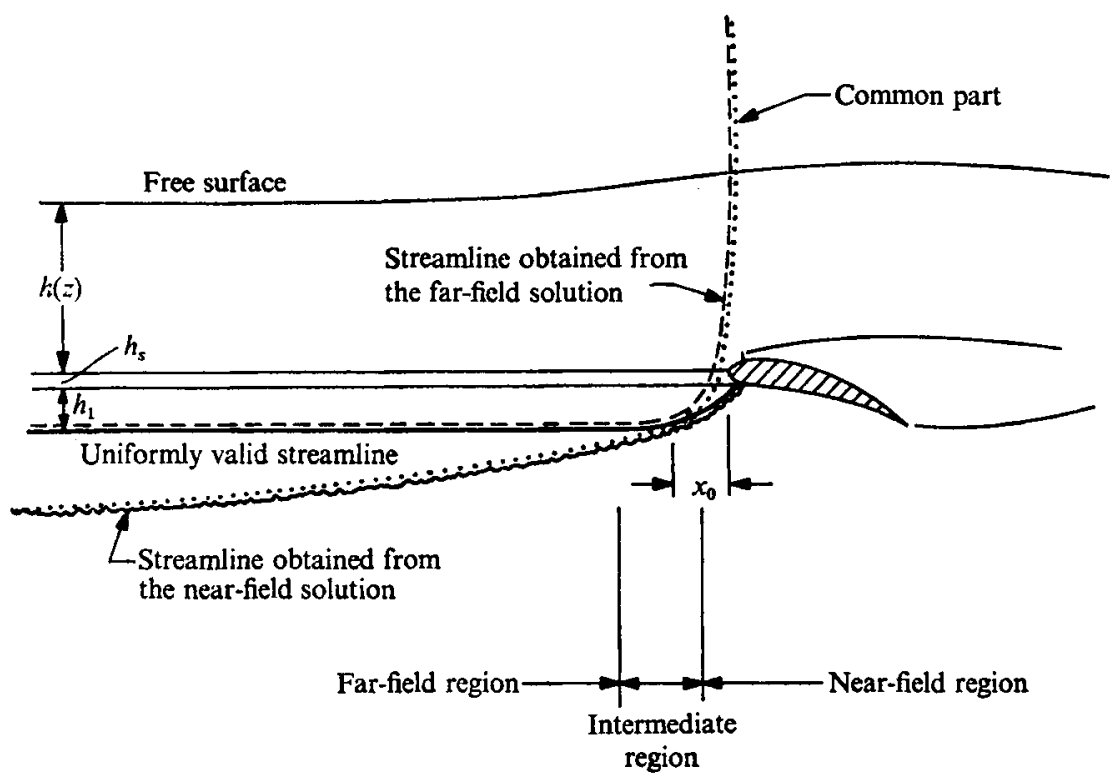

FIGUre 5. Determination of the submergence depth $h$, with the concept of the 'additive' composition method in the matching procedure.

that the flow angle experienced by the foil is equal to $-\alpha_{D}$. This is a well-known 'downwash' effect of the three-dimensional lifting foil, due to the trailing vortices first explained by Prandtl's lifting-line theory.

It is also seen that, even for the present cavity flow, no source-like terms appear in the $\Phi$ of the inner solution during the matching procedure, being consistent with the $\phi$ of the outer solution (1).

\subsection{Submergence depth $h(z)$}

The present problem incorporates the submergence depth $h(z)$ through the calculation of the location of the stagnation streamline. As has already been mentioned, the stagnation streamline of the near-field solution drops off logarithmically far upstream. This is depicted in figure 5 by the wiggling line. On the other hand, the same streamline calculated from the far-field solution by integrating the velocity in the $y$ direction shows an anomalous behaviour near the foil, again logarithmic, but this time like $\ln x$ as $x \rightarrow 0$, owing to the vortex singularity itself. At upstream infinity, however, this streamline has a definite reference level, to be used here. This situation is well suited to the application of a so-called additive composition method, to determine the location of the stagnation streamline and subsequently, the submergence depth $h$. This method is achieved by summing the near-field and far-field solutions, then correcting them by subtracting the part they have in common, the method being described in detail by Van Dyke (1964a). In the present problem, the elevation of the stagnation streamline can be calculated, to order $\epsilon^{2}$, by

$$
h_{1}=h_{i}+h_{0}-h_{c}
$$


where $h_{i}$ and $h_{0}$ are the elevated heights of the streamlines going from upstream infinity to the stagnation point obtained from the inner and outer solutions, respectively, and $h_{c}$ is that of the common part. Each term can be calculated as follows. Since $d Y=\operatorname{Im}\{\exp (-i \omega) d W\}$ from (3),

$$
h_{i}=\left.\epsilon \int_{-\infty}^{0} \operatorname{Im}\{\exp [i \omega(\zeta(\Phi))]\}\right|_{\Psi=0} d \Phi, \quad h_{0}=\left.\int_{-\infty}^{0} \frac{\partial \phi}{\partial y}\right|_{y=0} d x
$$

where $\phi$ in (1) is to be used, and

$$
h_{c}=\left.\int_{-\infty}^{0} v_{c}\right|_{y=0} d x
$$

where $v_{c}$ is the velocity of the common part in the $y$ direction, which has already been obtained during the matching procedure (i.e. from (13) or (22)),

$$
\left.v_{c}\right|_{y=0}=-\frac{\gamma(z)}{\pi} \frac{1}{x}+R_{0}
$$

where (24) has been applied. It is noted that (29) has logarithmic behaviour at both the origin and infinity. This is exactly the desired form for the common part to be subtracted from $h_{i}$ and $h_{0}$. The streamline of the common part is depicted in figure 5 by a dotted line going along the wiggling line for large negative $x$, and by the broken line near the foil. With the concept of the additive composition method applied, we can schematically illustrate a 'uniformly' valid streamline, now described by the thick bold line in the figure.

Substitution of (26)-(28) into (25) yields

$$
h_{1}=\left.\epsilon \int_{-\infty}^{0} \operatorname{Im}\{\exp [i \omega(\zeta(\Phi))]\}\right|_{\Psi=0} d \Phi+\left.\int_{-\infty}^{0} \frac{\partial \phi}{\partial y}\right|_{y=0} d x-\int_{-\infty}^{0}\left(-\frac{\gamma(z)}{\pi} \frac{1}{x}+R_{0}\right) d x
$$

Now the submergence $h(z)$ is expressed by making use of $\epsilon \Psi_{0}^{\circ}$, the total flow rate between the free surface and stagnation streamline:

$$
q_{\infty}\left(h+h_{S}+h_{1}\right)=\epsilon \Psi_{0},
$$

where $h_{S}$ is the vertical distance between the leading edge and stagnation point (as yet unknown). It should be mentioned that, in the actual calculation of (31), we do not carry out the integrations in $h_{i}, h_{0}$ and $h_{c}$ from infinity to zero, which leads to a computational difficulty of subtracting infinity from infinity. Instead, as also shown in figure $5, h_{1}$ is obtained by integrating the kernel of $(26)$ from $x_{0}$ to 0 , and (27) from $-\infty$ to $x_{0}$. The common part is not used except for finding $x_{0}$, which is an $x$ co-ordinate in the 'intermediate' region where the matching of the solutions has taken place. The alternative equation to (31) is then written as

$$
f_{4} \equiv \epsilon \Psi_{0}-\left\{h+h_{S}+\left.\epsilon \int_{\Phi_{0}\left(x_{0}\right)}^{0} \operatorname{Im}\{\exp [i \omega(\zeta(\Phi))]\}\right|_{\Psi=0} d \Phi+\left.\int_{-\infty}^{x_{0}} \frac{\partial \phi}{\partial y}\right|_{y=0} d x\right\}=0
$$

where $x_{0}$ is determined as a co-ordinate with which the vertical velocities of the inner and outer solutions, equivalently the common part and outer solution, match each other to order $\epsilon^{2}$. Thus,

$$
\left|v_{0}\left(x_{0}, z\right)-v_{c}\left(x_{0}\right)\right|=O\left(\epsilon^{2}\right),
$$


where, from (1),

$$
v_{0}(x, z)=\left.\frac{\partial \phi}{\partial y}\right|_{y=0}=\frac{1}{2 \pi} \int_{-1}^{1} \gamma\left(z^{\prime}\right) d z^{\prime} \frac{1}{\left(z-z^{\prime}\right)^{2}}\left(1+\frac{x}{\left[x^{2}+\left(z-z^{\prime}\right)^{2}\right]^{\frac{1}{2}}}\right) .
$$

As for the first integration in (32), since $\omega$ in the kernel is expressed in terms of $\zeta$ in $(6)$, it is easier to carry out the integration in the $\zeta$ plane by rewriting

$$
\left.\epsilon \int_{\Phi_{0}\left(x_{0}\right)}^{0} \operatorname{Im}\{\exp [i \omega(\zeta(\Phi))]\}\right|_{\Psi^{\prime}=0} d \Phi=\epsilon \int_{I}\left[\operatorname{Im}\left\{\exp \left[i \omega\left(\zeta^{\prime}\right)\right]\right\} \frac{d W}{d \zeta^{\prime}}\right] d \zeta^{\prime}
$$

where $I$ denotes the path of integration going from $\zeta_{0}\left(x_{0}\right)$ to the origin in the $\zeta$ plane, corresponding to the stagnation streamline. The path $I$ in terms of $\zeta$ will be found from (5) setting $\Psi=0$, and the point $\zeta_{0}$ that corresponds to $x_{0}$ can be determined by the formula, obtained from (3),

$$
x_{0}-x_{S}=\epsilon \operatorname{Re}\{Q\}=\left[\epsilon \operatorname{Re} \int_{0}^{\zeta_{0}} \exp \left[i \omega\left(\zeta^{\prime}\right)\right] \frac{d W}{d \zeta^{\prime}} d \zeta^{\prime}\right]
$$

where $x_{S}$ is the $x$ co-ordinate of the stagnation point. Finally, it is worthwhile to show that the last term in (32) can be written, using (34), as

$$
\left.\int_{-\infty}^{x_{0}} \frac{\partial \phi}{\partial y}\right|_{y=0} d x=\frac{1}{2 \pi} \int_{-1}^{1} \frac{\gamma\left(z^{\prime}\right) d z^{\prime}}{\left(z-z^{\prime}\right)^{2}}\left\{x_{0}+\left[x_{0}^{2}+\left(z-z^{\prime}\right)^{2}\right]^{\frac{1}{2}}\right\}
$$

\subsection{A system of nonlinear functional equations}

The last equation $f_{5}$ of the five unknown parameters is found by the assumption that the flow angle of the cavity sheet at downstream infinity is

$$
\operatorname{Im}\{\omega(d)\}=\alpha_{D}
$$

In the two-dimensional analysis, the flow angle there was chosen to be zero (see Furuya 1975), whereas it seems more reasonable to take this value to be the downwash angle $\alpha_{D}$ in the present three-dimensional problem. The above equation is explicitly written, using (6), as

$$
\begin{aligned}
f_{5} & \equiv \frac{\ln (1+\sigma)}{2 \pi}\left\{\ln \frac{(1+b)(a-d)}{2[(a+1)(a-1)(d+1)(d-b)]^{\frac{1}{2}}+(a+1)(d-b)+(a-b)(d+1)}\right. \\
& \left.+\ln \frac{(1+b)(d-c)}{2[(c+1)(c-b)(d+1)(d-b)]^{\frac{1}{2}}+(c+1)(d-b)+(c-b)(d+1)}\right\} \\
& -\frac{[(d+1)(d-b)]^{\frac{1}{2}}}{\pi} \int_{-1}^{b} \frac{\beta\left(\xi^{\prime}\right)}{\left[\left(1+\xi^{\prime}\right)\left(b-\xi^{\prime}\right)\right]^{\frac{1}{2}}} \frac{d \xi^{\prime}}{\xi^{\prime}-d}+\left(\frac{\pi}{2}+\sin ^{-1} \frac{d(b-1)+2 b}{d(b+1)}\right)-\alpha_{D}=0 .
\end{aligned}
$$

With this, the mathematical formulation is completed. This consists of a system of nonlinear functional equations, $f_{1}$ to $f_{5}$ in (7), (12), (24), (32) and (38), for the solution parameters $a$ to $d$ and $\Psi_{0}$, all being functions of the spanwise co-ordinate $z$, with an additional equation (23) for $\gamma(z)$. In $\$ 3$ a numerical method to solve this system will be discussed in detail. 


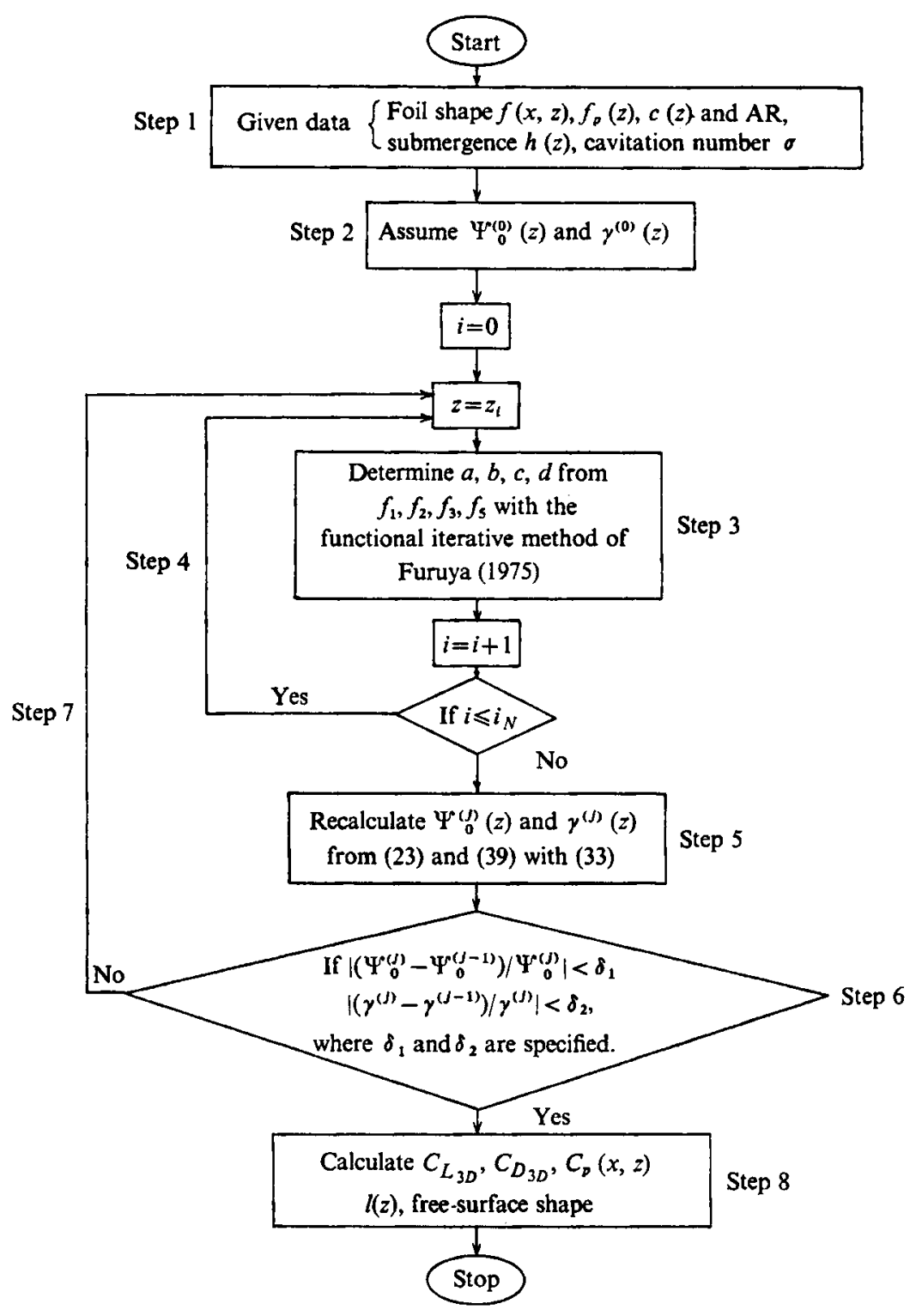

Figure 6. Flow chart describing the numerical iterative procedure in solving the system of the nonlinear functional equations.

\section{Numerical procedure for a system of nonlinear functional equations}

The system of nonlinear functional equations here is similar to that of Furuya (1975), except that one more unknown parameter $\Psi_{0}$ is added, and all of the parameters are functions of the spanwise co-ordinate $z$. In order to use the solution procedure of that work, $\gamma(z)$ and $\Psi_{0}$, as yet unknown functions, are to be assumed to initiate the iterative calculation. In what follows, the detailed computational procedure will be explained step by step using the flow chart in figure 6 . 
With a set of a complete foil profile such as $y=f(x, z), x_{p}=f_{p}(z), c(z)$, aspect ratio, submergence depth $h(z)$, cavity separation points and cavitation number $\sigma$ specified as by step 1 in figure 6, we start two-dimensional sectional computations, working on just four equations, $f_{1}, f_{2}, f_{3}$ and $f_{5}$ with assumed values of $\Psi_{0}$ and $\gamma$, leaving $f_{4}$ temporarily unused (step 2). If $\Psi_{0}$ and $\gamma$ are known, these four equations are exactly the same as those of Furuya (1975). In this way, the relation over the spanwise direction is decoupled to determine $a$ to $d$ at each spanwise control point by using the functional iterative method (step 3). Finding $a$ to $d$ at discrete control points (step 4; the number of the points is denoted by $i_{N}$ in figure 6), $\gamma$ and $\Psi_{0}$ are to be revaluated, respectively, by (23) and a modification of $(32)$, i.e.

$$
\begin{aligned}
\Psi_{0}^{(j+1)}=\left(h+h_{S}\right)\left\{\epsilon-\frac{1}{\Psi_{0}^{(j)}}\right. & {\left[\epsilon \int_{I}\left[\operatorname{Im}\{\exp [i \omega(\zeta)]\} \frac{d W}{d \zeta}\right] d \zeta\right.} \\
& \left.+\frac{1}{2 \pi} \int_{-1}^{1} \frac{\gamma\left(z^{\prime}\right) d z^{\prime}}{\left(z-z^{\prime}\right)^{2}}\left(x_{0}+\left[x_{0}^{2}+\left(z-z^{\prime}\right)^{2}\right]^{\left.\frac{1}{2}\right)}\right]\right\}^{-1}
\end{aligned}
$$

where (35) and (37) have been used ( $\operatorname{step} 5) . h_{S}$ in this equation is set to zero at the first iteration loop (where $j=1$ ), whereas calculated values of $h_{S}$ will be incorporated in the successive steps. (Actually, $h_{S}$ is negligibly small as long as overall body angles of the foil are relatively small.) The choice of the number, as well as of the location, of control points over the span is arbitrary, but usually more points are taken near the tip of the foil, where $\gamma$ is expected to change abruptly. A numerical example of this procedure used in subsequent calculations is that with $z_{n}=-\cos \theta_{n}$ and $\theta_{n}=\frac{1}{8} n \pi$, where $n=1-4$ is taken.

Although values of $\gamma$ are evaluated only at discrete points, they are interpolated by the Fourier sine series

$$
\gamma(z)=\sum_{n=1}^{i_{N}} A_{n} \sin n \theta, \quad z=-\cos \theta
$$

for the subsequent iteration loops. This expression is very useful, in that $\alpha_{D}$ in (24) is now given in a closed form,

$$
\alpha_{D}=\frac{1}{2 \pi} \int_{0}^{\pi} \frac{\sum_{i=n}^{i_{N}} n A_{n} \cos n \theta}{\cos \theta-\cos \theta_{1}} d \theta=\frac{1}{2} \sum_{n=1}^{i_{N}} n A_{n} \frac{\sin n \theta_{1}}{\sin \theta_{1}}, \quad z=-\cos \theta_{1}, \quad(41 a, b)
$$

and also in that the averaged circulation over the $\operatorname{span} \gamma_{\mathrm{av}}$ is easily calculated,

$$
\gamma_{\mathrm{av}}=\frac{1}{\text { (span length) }} \int_{-1}^{1} \gamma\left(z^{\prime}\right) d z^{\prime}=\frac{1}{4} \sum_{n=1}^{i N} A n\left[\frac{\sin (n-1) \pi}{n-1}-\frac{\sin (n+1) \pi}{n+1}\right] \text {, }
$$

where the span length has been taken to be two.

In the next step, step 6 , the values of

$$
\left|\left(\Psi_{0}^{(j+1)}-\Psi_{0}^{(j)}\right) / \Psi_{0}^{(j)}\right| \text { and }\left|\left(\gamma^{(j+1)}-\gamma^{(j)}\right) / \gamma^{(j)}\right|
$$

are calculated, to check if the system reaches a convergent state within a required accuracy. If not, we go back to the original point and repeat the same procedure until the system converges (step 7). Finally, three-dimensional force coefficients 
$C_{L_{3 D}}$ and $C_{D_{3 D}}$, sectional pressure coefficients $C_{p}(x, z)$, cavity shapes and lengths and free-surface shapes are computed in step 8 .

It may be mentioned that the present iterative procedure has been found to provide a very fast and stable convergent solution for a rectangular flat-plate foil: e.g. the number of iterations from 4 to 8 was sufficient for the system to converge, with the relative errors mentioned above all less than $3 \times 10^{-3}$.

\section{Basic flow characteristics}

The overall lift and drag coefficients $C_{L_{3 D}}$ and $C_{D_{3 D}}$ are obtained by integrating the local $C_{L}$ and $C_{D}$ over the span. An alternative way to find $C_{L_{3} D}$, as well as the local $C_{L}$ 's, is to make use of $(42)$ with $\gamma(z)$ given by $(23)$, since we have the relation between $C_{L}$ and $\gamma$

$$
C_{L}=\frac{\rho q_{\infty} \gamma}{\frac{1}{2} \rho q_{\infty}^{2} \text { chord }}
$$

It is very convenient to have two ways of calculating the lift coefficient independently, specifically because the numerical computations as well as the theoretical basis can be checked.

\section{Numerical results for rectangular flat plates}

A flat-plate hydrofoil of rectangular planform with no dihedral angle is chosen as an example of the numerical computation. With the angle of the body $\alpha(=-\beta$ in the definition of figure 3) taken to be constant, the integrations involving $\beta$ in step 3 of the numerical procedure are reduced to simple closed forms. For the starting values of $\epsilon \Psi_{0}^{(1)}$ and $\gamma^{(1)}$, we have used, respectively, $h$ itself and a fully wetted rectangular flat foil (given in e.g. Glauert 1948), with the value estimated from $C_{L_{2 D}}$, which can be calculated for the same $\alpha, \sigma$ and $\Psi_{0}$.

Figures 7-9 show three-dimensional lift and drag coefficients against the cavitation number for the aspect ratios $2 \cdot 5,4$ and 6 , respectively, at various angles of attack. The present theoretical results are in an excellent agreement with the experimental data of Schiebe \& Wetzel (1961). Although the present theory has assumed large aspect ratio, short cavity and shallow submergence, the numerical results compare well with the experimental data even for an aspect ratio $2 \cdot 5, \sigma=0 \cdot 1$ and $h=2$ chords. But it was expected that the present theory predicts the flow very well for large angles of attack, even up to $20^{\circ}$, since the nonlinear exact theory is employed. The only theoretical data available for comparison at a finite cavitation number in the presence of a free surface are those of Nishiyama \& Miyamoto (1969) at $\mathrm{AR}=6$ and $\alpha=8.5^{\circ}$. These are shown in figures $9(a),(c)$. They compare well with the present results. It is not known, however, why their results, made with fully linearized lifting-surface theory, provide values smaller than the present nonlinear results. (Usually linear theories over-estimate forces, as described by Wu (1959). Even an $8.5^{\circ}$ angle of attack for a two-dimensional flat-plate foil, at $\sigma=0 \cdot 1$, is sufficient to cause an error of $12 \%$, for example.) The theoretical data for $\sigma=0$ by Johnson (1957) are also plotted in 

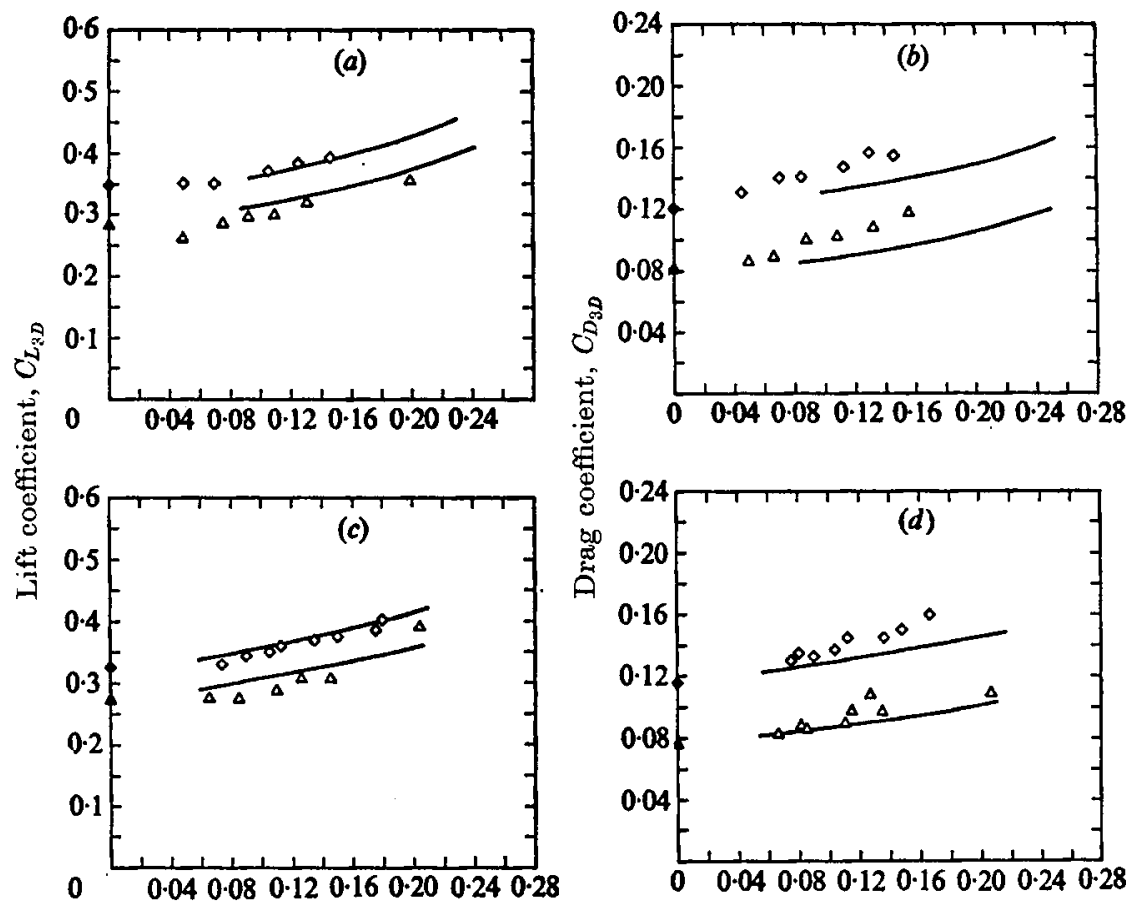

Cavitation number, $\sigma$

FIGURE 7. Three-dimensional lift and drag coefficients $C_{L_{3 D}}$ and $C_{L_{3 D}}$ against cavitation number $\sigma$, for aspect ratio $2 \cdot 5$. ___ ; present theory; $\Delta, \diamond$, experimental data of Schiebe \& Wetzel (1961) for $\alpha=16$ and $20^{\circ}$, respectively; $\Delta, \Delta$, theoretical data of Johnson (1957) for $\sigma=0$, and $\alpha=10$ and $14^{\circ}$, respectively. $(a),(b)$ Submergence depth $h=1$ chord; $(c),(d)$ $h=2$ chords.
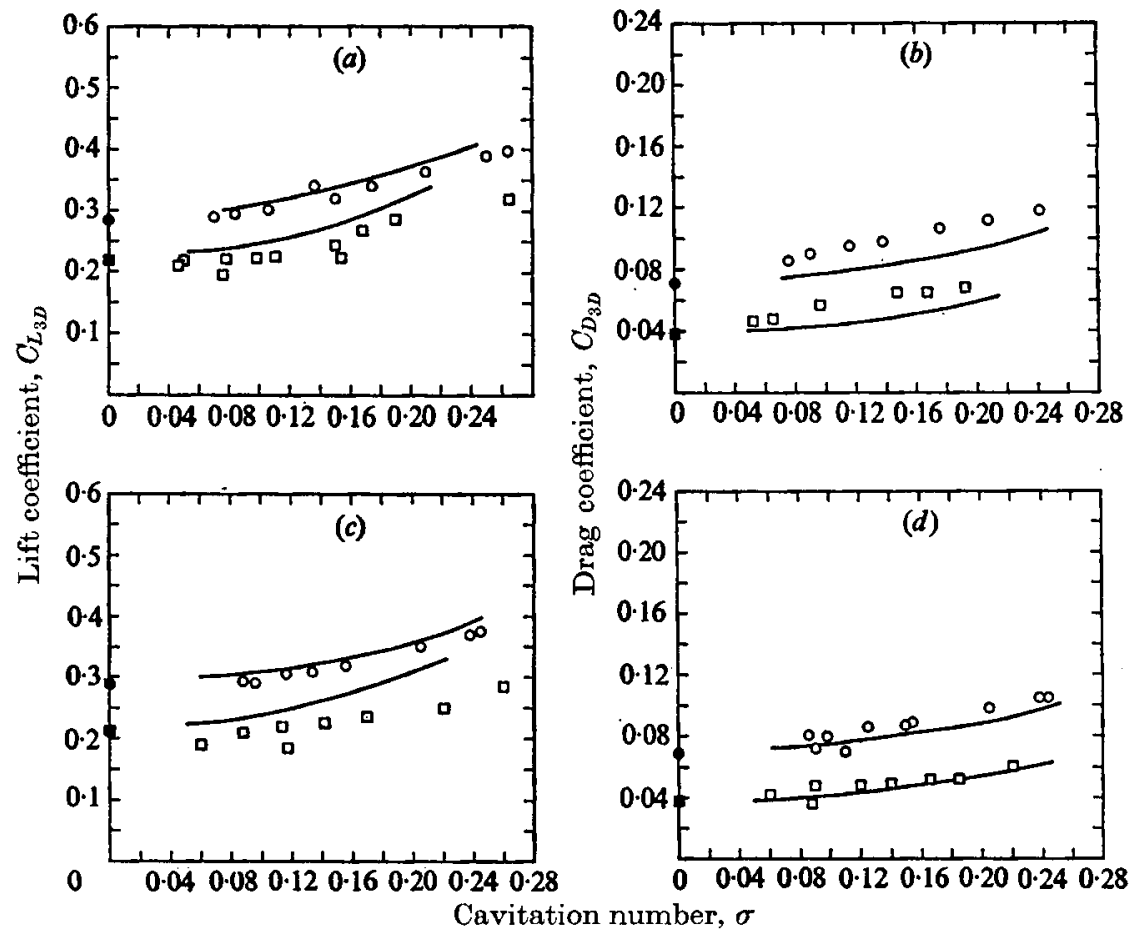

FIGURE 8. $C_{L_{3 D}}$ and $C_{D_{3 D}}$ against $\sigma$, for aspect ratio 4. - , present theory; $\square$, $\bigcirc$, experimental data of Schiebe \& Wetzel (1961) for $\alpha=16$ and $20^{\circ}$, respectively; $\square$, theoretical data of Johnson (1957) for $\sigma=0$, and $\alpha=10$ and $14^{\circ}$, respectively. $(a),(b)$ Submergence depth $h=1$ chord; $(c),(d) h=2$ chords. 

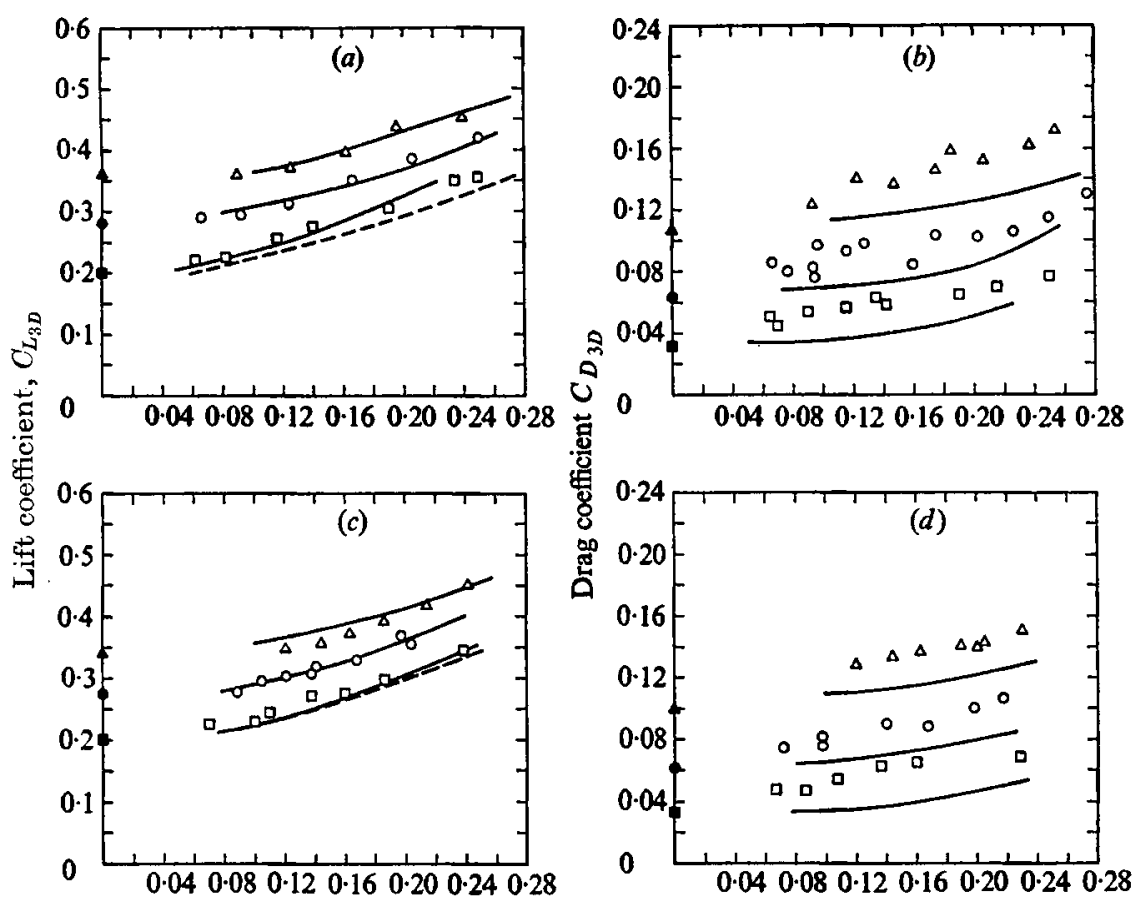

Cavitation number, $\sigma$

Figure 9. $C_{L_{3 D}}$ and $C_{D_{3 D}}$ against $\sigma$, for aspect ratio $6 .-\ldots$, present theory; $\square, \bigcirc, \triangle$, experimental data of Schiebe \& Wetzel (1961) for $\alpha=8.5,12.5$ and $16.5^{\circ}$, respectively; $\square, \Delta$, theoretical data of Johnson (1957) for $\sigma=0$ and $\alpha=8.5,12.5$ and $16 \cdot 5^{\circ}$, respectively; ----, theoretical data of Nishiyama \& Miyamoto (1969) for $\alpha=8 \cdot 5^{\circ}$. (a), (b) Submergence depth $h=1$ chord; $(c),(d) h=2$ chords.

each figure, showing the same trend of the present curves as the limiting case of $\sigma$ approaching zero. The calculated three-dimensional drag coefficients $C_{D_{3 D}}$ are on the whole smaller than those of the experiment, the discrepancy possibly being attributable to the viscous frictional forces and the downwash effect of supporting struts in the experiment.

The effect of the aspect ratio on $C_{L_{3 D}}$ and the correction factor $K=C_{L_{3 D}} / C_{L_{2 D}}$ is shown in figure 10 for three different cases. The results show not much difference in $K$ for different values of $\sigma$ and $h$, whereas the change in angle of attack $\alpha$ has generated a considerable amount of shift of one $K$ curve from the others. The present $C_{L_{2 D}}$ 's were calculated using the nonlinear exact theory, with an averaged $\Psi_{0}$ obtained from three-dimensional calculations. In the same figure, the correction factors obtained by the lifting-line theory of Prandtl for a fully wetted foil of elliptic loading, by the same theory applied to a cavitating flow by Nishiyama (1970), and by a lifting-surface theory by Nishiyama \& Miyamoto (1969), all in an unbounded flow medium, are shown for comparison. Among them, the last theory, although fully linearized, compares well with the present result, in spite of the inconsistent $C_{L_{8 D}}$ predicted by this theory.

In figure 11 the effect of submergence depth on $C_{L_{3 D}}$ is shown for two cases. The 

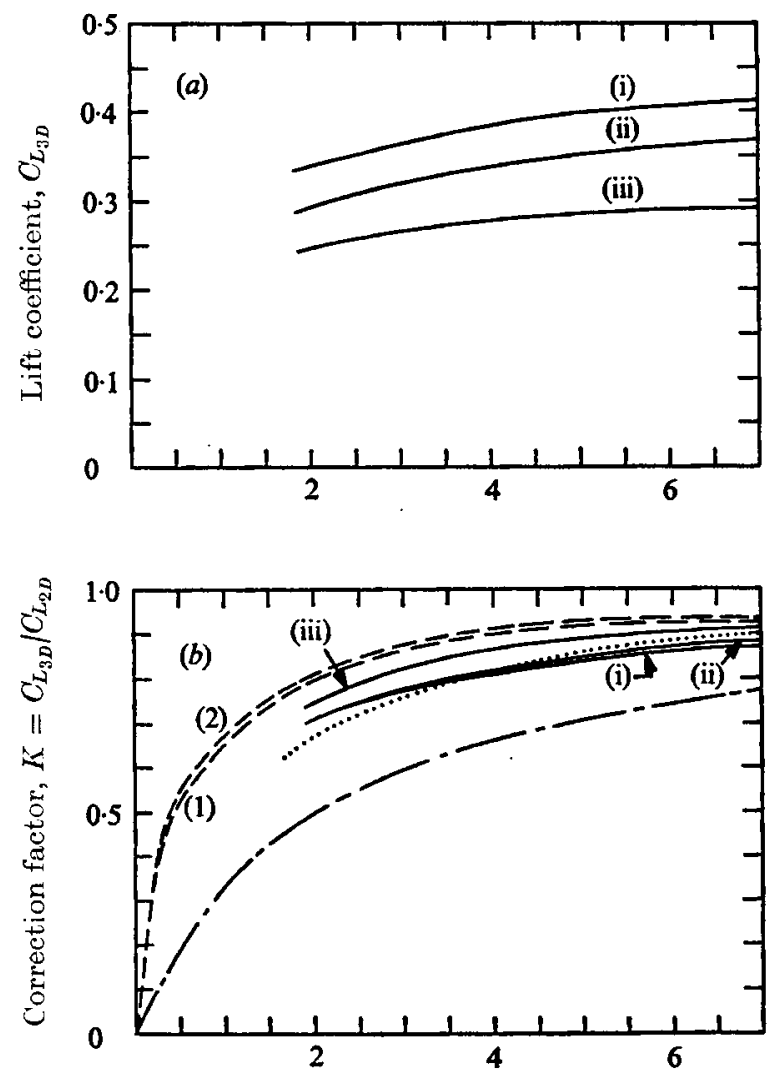

Aspect ratio

FIGURE 10. Effect of aspect ratio on $(a) C_{L_{3 D}}$ and (b) correction factor $K=C_{L_{3 D}} / C_{L_{2 D}}$. $\longrightarrow$, present theory; - - - - lifting-line theory of Nishiyama (1970), for (1) $\sigma / \alpha=1 \cdot 18$, (2) $\sigma / \alpha=0.69$, at $h=\infty ; \cdots$, by lifting-surface theory of Nishiyama \& Miyamoto (1969), for $\sigma=1 \cdot 18, h=\infty$; - - - - Prandtl, for a fully wetted foil of elliptic loading at $h=\infty$.

$\begin{array}{ccccc} & \alpha \text { (degrees) } & \sigma & h \text { (chords) } & C_{L_{2 D}} \\ \text { (i) } & 16 \cdot 0 & 0 \cdot 18 & 1 & 0 \cdot 471 \\ \text { (ii) } & 16.0 & 0 \cdot 10 & 2 & 0.415 \\ \text { (iii) } & 8.5 & 0 \cdot 18 & 2 & 0.320\end{array}$

trend of increase in the lift coefficients as the submergence becomes smaller is seen for both cases.

For $h$ less than about 0.5 chords and for small $\sigma$ the present numerical analysis faces a computational difficulty, since the two solution parameters $c$ and $d$ become almost identical up to seven digits. The single-precision computations presently used are not sufficiently accurate to detect the difference, so that we should use double precision for these cases.

In figure 12 the distribution of local lift coefficients is given for three different cases, showing the tendency that the smaller the aspect ratio, the more reduction of local $C_{L}$ near the tip. The detailed flow configurations, for a representative flow case of $\alpha=14^{\circ}, \sigma=0 \cdot 18$, aspect ratio 4 and $h=1$ chord, are shown in figure 13, 


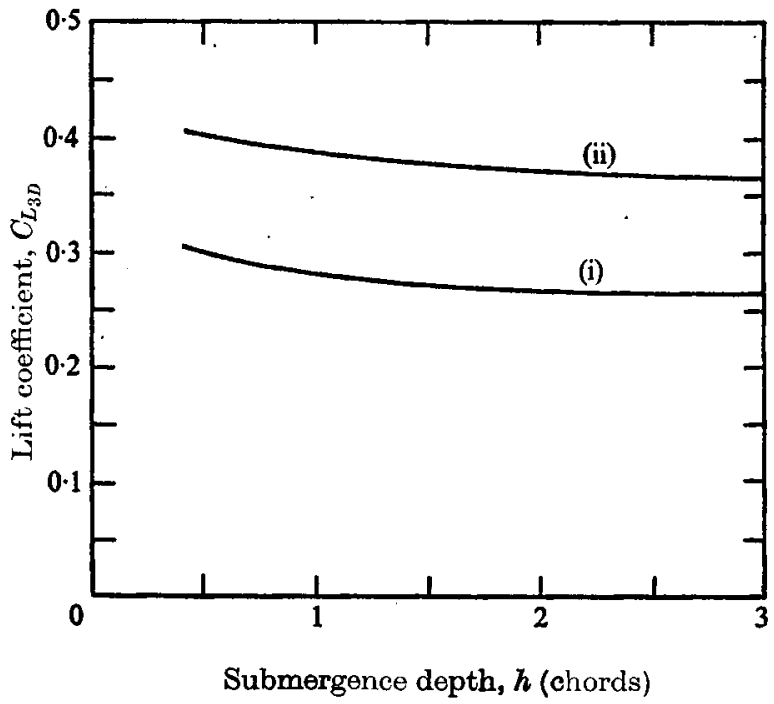

FigURE 11. Effect of submergence depth of rectangular flat plate on $C_{L_{\mathbf{z} D}}$. Aspect ratio $\quad \alpha$ (degrees)

$0 \cdot 18$

(i) $6 \cdot 0$

$8 \cdot 5$

0.22

(ii)

$4 \cdot 0$

$14 \cdot 0$

$0 \cdot 22$

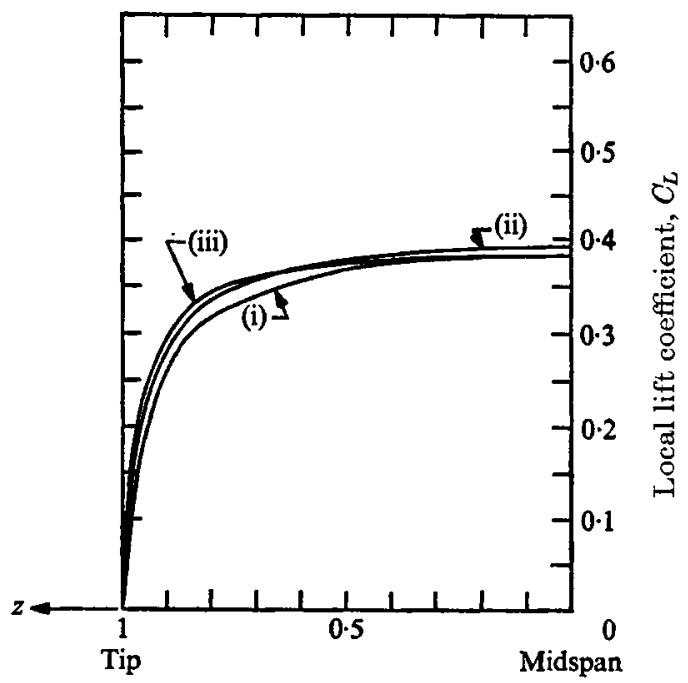

Figure 12. Local distribution of the lift coefficients over the spanwise direction.

Aspect ratio $\quad \alpha$ (degrees)

(ii)

(iii)
$2 \cdot 5$

$4 \cdot 0$

$6 \cdot 0$
$16 \cdot 0$

$14 \cdot 0$

$12 \cdot 5$ $\sigma$

$0 \cdot 18$

0.18

$0 \cdot 18$ $h$ (chords)

2
1
1



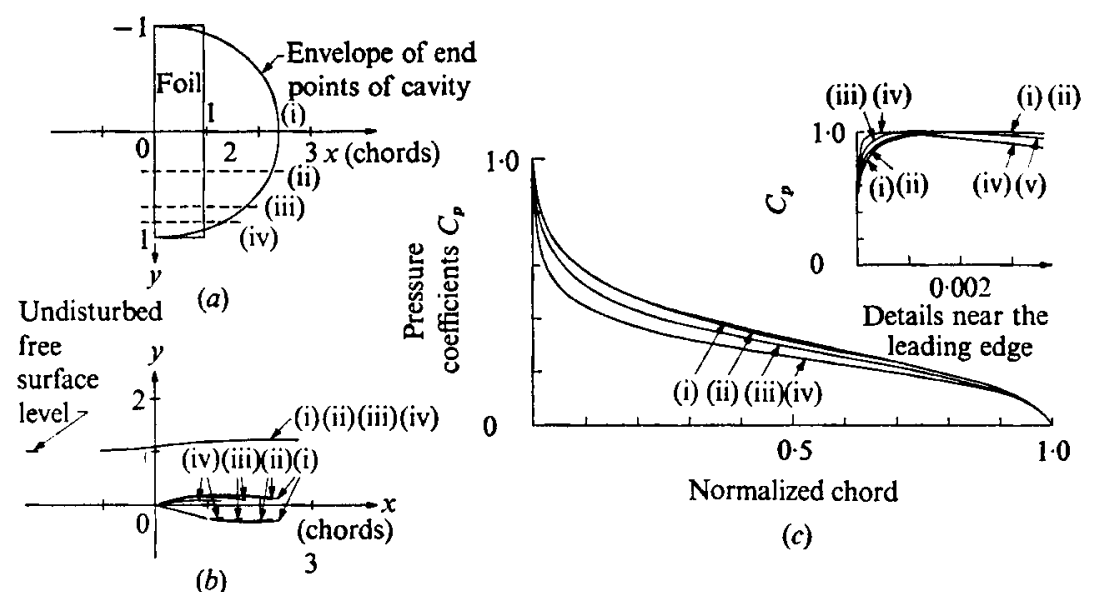

(b)

(c)

Figure 13. (a), (b) cavity and free-surface shapes for a rectangular flat-plate hydrofoil with $A R=4, \alpha=14^{\circ}, \sigma=0.18$ and $h=1$ chord, together with (c) the local pressure coefficients $C_{p}$ at four different spanwise locations, these $z$ co-ordinates being shown in $(a)$. (i) $y=0$. (ii) $y=0.382$. (iii) $y=0.707$. (iv) $y=0.858$.

which describes $(a),(b)$ the length and shape of the cavity and free-surface location, and $(c)$ local pressure distributions in terms of $C_{p}=\left(p-p_{c}\right) / \frac{1}{2} \rho q_{c}^{2}$, together with magnified $C_{p}$ near the leading edge. The free-surface shape is nearly invariant in the spanwise direction, although there exists an unnoticeable hump, within the scale used here, near the location of (iii), possibly due to the rolling-up effect of the tip.

The present numerical computations were made on the IBM 370-158 in the Computer Center at the California Institute of Technology. The convergence of the numerical iterative procedure proposed here has been found to be fast and stable, in most cases requiring four to eight iteration loops. The maximum execution time for one data point was about $200 \mathrm{~s}$.

\section{Conclusion}

The present method, consisting of the nonlinear exact two-dimensional freestreamline theory and Prandtl's lifting-line theory, shows excellent agreement with the experimental data, even for cases of a low aspect ratio (e.g. 2-5), small cavitation number and larger angles of attack.

Although present numerical computations have been made only for a rectangular flat plate, the method may be used for an arbitrary planform and foil profile, including a rounded leading edge and a small dihedral angle, although, in these cases, more computational time would be required. We anticipate no difficulties whatsoever in obtaining convergent solutions, as the most difficult part of the computation is in well-developed two-dimensional flow.

The author would like to thank Professor A.J. Acosta for his helpful advice and continuing interest. Discussions with Dr Y. Shen are also appreciated. This 
work was supported by the Naval Ship Systems Command General Hydrodynamics Research Program, technically administered by the Naval Ship Research and Development Center, under contract N00014-67-A-0094-0031.

\section{REFERENCES}

Acosta, A. J. 1968 Two models for cavity flow, a theoretical summary and application. J. Basic Engng, Trans. A.S.M.E. D 90, 634.

Acosta, A. J. 1973 Hydrofoils and hydrofoil craft. Ann. Rev. Fluid Mech. 5, 161-184.

Cole, J. D. 1968 Perturbation Methods in Applied Mathematics. Blaisdel.

FurUyA, O. 1975 Nonlinear calculation of arbitrarily shaped supercavitating hydrofoils near a free surface. $J$. Fluid Mech. 68, 21-40.

GLAuert, H. 1948 The Elements of Aerofoil and Airscrew Theory. Cambridge University Press.

Green, A. E. 1936 Note on the sliding of a plate on the surface of a stream. Proc. Camb. Phil. Soc. 32, 248-252.

Jornson, V. E. 1957 Theoretical and experimental investigation of arbitrary aspect ratio, supercavitating hydrofoils operating near the free surface. N.A.C.A., R.M., LS 7116.

Larock, B. \& Street, R. 1967 Nonlinear solution for a fully cavitating hydrofoil beneath a free surface. J. Ship Res. 11, 131-140.

LEeHEy, P. 1971 Supercavitating hydrofoil of finite span. IUTAM Symp., Leningrad, pp. 277-299.

Nishiyama, T. 1970 Lifting line theory of supercavitating hydrofoil of finite span. Z. angew. Math. Mech. 50, 645-653.

NishiYana, T. \& Mryamoto, M. 1969 Lifting-surface method for calculating the hydrodynamic characteristics of supercavitating hydrofoil operating near the free water surface. Tohoku University, Tech. Rep. 34, 2, 123-139.

Schiebe, F. R. \& Wetzer, J.M. 1961 Ventilated cavities on submerged three-dimensional hydrofoils. St Anthony Falls Hydraulic Lab., University of Minneapolis, Tech. Paper, B 36.

Shen, Y. T. \& OGILvIE, T. F. 1972 Nonlinear hydrodynamic theory for finite-span planing surface. J. Ship Res. 16, 3-21.

Tuliv, M. P. 1964 Supercavitating flows : small perturbation theory. J. Ship Res. 7, 16-37.

VAN Dyke, M. 1964 a Perturbation Methods in Fluid Mechanics. Academic.

VAN Dyke, M. $1964 b$ Lifting-line theory as a singular perturbation problem. J. Appl. Math. Mech. 28, 90-101.

Widnald, S. E. 1966 Unsteady loads on supercavitating hydrofoils of finite span. J. Ship Res. 10, 107-118.

Wv, T. Y. 1959 A note on the linear and nonlinear theories for fully cavitated hydrofoils. Hydrodynamic Lab., Calif. Inst. of Tech., Rep. no. 21-22. 